092

\section{CONFRONTO TRA SISTEMI AUTOMATICI NELLA DETERMINAZIONI SIEROLOGICHE DI HBV, HCV E HIV}

\author{
De Rango C., Gianello R., Dusi D., Molinari C., Faccoli R. \\ Servizio di Medicina di Laboratorio, \\ Fondazione Poliambulanza Istituto Ospedaliero, Brescia.
}

Introduzione e scopo del lavoro. Abbiamo inteso confrontare le prestazioni analitiche di due sistemi automatizzati con lettura in chemiluminescenza (Architect, Abbott Diagnostics e Vitros Eci, Ortho Clinical Diagnostics) per le analisi dei marcatori sierologici di infezione da virus HBV, HCV e HIV. Metodi. Campioni di "routine" $(n=24)$ sono stati analizzati in parallelo con i due stistemi. I campioni reattivi per HBsAg, antiHCV o anti-HIVsono stati analizzati con test di secondo livello. Risultati.

A) HBsAg: su 224 campioni la concordanza era 99,1\%, con una migliore specificità Architect (100\% vs. 99,45\%). Abbiamo analizzato la correlazione tra il segnale Architect e quello Vitros su 21 campioni positivi, trovando una relazione lineare $(\mathrm{r}=0,976)$.

B) Anti-HCV: 224 campioni, concordanza 95,1\%, 11 discrepanti di cui 10 reattivi Vitros e negativi Architect (6 negativi e 4 indeterminati al RIBA), per una specificità del 98,7\% con Architect e del 91,8\% con Vitros.

C) Anti-HIV: 106 campioni, concordanza 95,3\%; i 5 discrepanti (tutti reattivi Vitros e negativi Architect) erano falsi positivi: sui 4 positivi sia con Architect che con Vitros, due erano positivi per antigene p24 con il test Vidas p24 Duo.

D) anti-HBs; su 41 campioni clinici la concordanza era quasi assoluta: l'unico discordante era debolmente positivo con Architect $(15,6 \mathrm{mUI} / \mathrm{mL})$ e negativo con Vitros ( $5 \mathrm{mUI} / \mathrm{mL}$ ). La correlazione tra i due test su 26 campioni era buona $(r=0,906)$, con i valori Architect più elevati. E) Altri marcatori HBV: concordanza quasi assoluta su 28 determinazioni tra $\mathrm{HBeAg}$, anti-HBe e anti-HBc.

Conclusioni. I test sierologici sul sistema Architect appaiono più specifici del corrispondenti test sul sistema Vitros, con delle importanti implicazioni gestionali e di spesa. Inoltre, la possibilità della determinazione simultanea di antigene p24 e anticorpi anti-HIV, garantita dal test Architect HIV Ag/Ab Combo, è di concreta utilità nella diagnosi precoce dell'infezione da HIV.

\section{3}

\section{CONFRONTO TRA DATABASE ONLINE PER LA SOTTOTIPIZZAZIONE DI SEQUENZE HVI-I}

Di Nicuolo G.', Battisti S.', La Porta R.', Starace M.', Gargiulo M. ${ }^{2}$,Viglietti R. ${ }^{2}$

'Servizio di Virologia,

23ㅡ Divisione Malattie Infettive, A. O. "D. Cotugno", Napoli

Introduzione. Le sequenze del gene pol di HIV-1 ottenute con il test di farmaco-resistenza sono anche usate per la sottotipizzazione. Scopo di questo lavoro è stato confrontare tre database online comunemente usati per la definizione del sot- totipo e delle forme circolanti ricombinanti (CRFs) di HIV-1. Materiali e metodi. Sono state incluse nello studio le sequenze del gene pol di HIV-1 di 442 soggetti arruolati per il test di farmaco-resistenza. Il sequenziamento è stato effettuato con kit ViroSeq ${ }^{\mathrm{TM}}$ HIV-1 Genotyping System (Celera Diagnostics, USA) su ABI Prism 377 DNA Sequencer (Applied Biosystem, USA). Le sequenze sono state sottoposte ad interpretazione per la definizione del sottotipo ai database Stanford://hivdb.stanford.edu/, NCBI://ncbi.nih.gov/, REGA://dbpartners.stanford.edu/RegaSubtyping/

Risultati. Di 442 sequenze: 397=B (89,9\%), 45 non-B $(10,2 \%)$, $\mathrm{i}$ tre i sistemi analitici hanno fornito risultati concordanti per $378(85,5 \%)$ : $\mathrm{B}=355(89,4 \%)$, non- $\mathrm{B}=23(37,8 \%)$. Una significativa discordanza $(\mathrm{p}<0.001)$ è stata osservata nella definizione dei sottotipi non-B. Stanford ha assegnato il sottotipo non-B in tutti i casi: 35 sottotipi o CRFs definiti e 10 misti (sottotipo o CRF differente per RT e PR); REGA solo in 37 casi come sottotipo o CRF definito (8 non assegnati); NCBI in 28 casi come sottotipo o CRF definito e in 17 misto ( 2 o più sottotipi/CRFs). I 23 risultati concordanti dei tre sistemi analitici per sottotipo non-B erano: CRF02_AG $=16$, $\mathrm{A}=3, \mathrm{C}=2, \mathrm{D}=1, \mathrm{G}=1$. Stanford e REGA hanno dato risultati concordanti in $n=31(68,9 \%)$ casi, Stanford ed NCBI in $n=23$ $(51,1 \%)$, REGA ed NCBI in $n=27(60,0 \%)$. Dei 22 risultati discordanti Stanford ne ha assegnati 12 come sottotipi o CRFs definiti e 10 misti, REGA 14 come sottotipi definiti e 8 $(17,8 \%)$ non assegnati, NCBI 4 definiti e 18 misti.

Conclusioni. Nell' $85 \%$ dei casi c'è una buona concordanza tra $\mathrm{i}$ tre approcci analitici. La concordanza è soddisfacente per il sottotipo B $(89,4 \%)$, mentre per i non-B si osserva una discordanza statisticamente significativa. L'applicazione alla pratica clinica di questi sistemi per la sottotipizzazione di HIV-1deve essere considerata con prudenza.

\section{4}

\section{SORVEGLIANZA}

\section{EPIDEMIOLOGICO-MOLECOLARE DEI VIRUS RESPIRATORI IN UNA COORTE DI SOG- GETTI PEDIATRICI (2004)}

\author{
Perin S.,' Tanzi E., ${ }^{2}$ Ruzza ML., ${ }^{3}$ Amendola A., ${ }^{2}$ Colzani D., ${ }^{2}$ \\ Podestà AF., Farina C.' \\ UO Microbiologia, \\ ¿UO Pediatria - AO 'Ospedale San Carlo Borromeo' - Milano e \\ 2Dipartimento di Sanità Pubblica - Microbiologia \\ - Virologia dell'Università degli Studi di Milano, Milano
}

Introduzione. La sorveglianza epidemiologico-molecolare dei virus respiratori nelle popolazioni a maggior incidenza di infezioni respiratorie acute $(A R I)$, quale quella pediatrica, è un elemento essenziale per predisporre strategie diagnostiche, terapeutiche e preventive sempre più mirate. Inoltre consente di correlare la manifestazione clinica all'assetto genetico dei virus coinvolti.

Materiali e metodi. Nel periodo marzo-maggio 2004 sono stati arruolati 108 soggetti di età pediatrica $(59 \mathrm{M}, 49 \mathrm{~F}$; età media: 32.1 mesi) con sintomatologia correlabile ad ARI. Ad ognuno di essi è stato effettuato un tampone oro-faringeo (TF) successivamente analizzato mediante l'applicazione di un pannello di saggi molecolari per la diagnosi virologica:

1) multiplex-PCR per la determinazione simultanea dei virus Influenzali A e B e dell'RSV; 
2) multiplex-PCR per i Coronavirus $229 \mathrm{E}$ e OC43;

3) multiplex-PCR per i virus Parainfluenzali 1-4 (HIPV 1-4);

4) emi-nested-PCR per la determinazione delle sequenze geniche dei Rhinovirus;

5) nested-PCR per la ricerca di sequenze geniche dei Metapneumovirus umani (HPMV).

Risultati. La diagnosi virologico-molecolare ha evidenziato, nel 49.1\% (53/108) dei campioni biologici analizzati la presenza di acido nucleico di uno o più degli agenti virali indagati. In particolare, i Coronavirus $(33.3 \%)$ e i Rhinovirus $(30 \%)$ erano $\mathrm{i}$ virus principalmente identificati $(\mathrm{p}<0.05)$, seguiti da HMPV (9.4\%), RSV (7.5\%), Influenza A e HIPV3 (5.7\%), Influenza B (3.8\%); nel 13.2\% sono state evidenziate co-infezioni virali.

Il 51.4\% delle infezioni delle alte vie respiratorie ed il 41.7\% delle infezioni delle basse vie respiratorie era associata ad una eziologia di tipo virale, con Rhinovirus e Coronavirus come agenti eziologici principalmente coinvolti nelle prime, mentre Coronavirus (40\%) e virus influenzali (26.6\%) nelle seconde. Conclusioni. I risultati ottenuti confermano la rilevanza dei virus come agenti eziologici delle ARI in età pediatrica, anche nel periodo primaverile. La caratterizzazione virologico-molecolare ha permesso sia di valutare la distribuzione epidemiologica stagionale dei virus associati alle ARI, sia di correlare patogeni e manifestazioni cliniche nei soggetti in età pediatrica.

\section{5}

\section{SORVEGLIANZA DELL'INFLUENZA IN ETÀ PEDIATRICA (2004-2005)}

\author{
Amendola A.,' Perin S., ${ }^{2}$ Ruzza ML., ${ }^{3}$ Farina C., ${ }^{2}$ Pariani E., \\ 'Podestà AF., ${ }^{3}$ Zappa A.', Tanzi E.' \\ 'Dipartimento di Sanità Pubblica - Microbiologia \\ - Virologia dell'Università degli Studi di Milano, Milano \\ ¿UO Microbiologia, ${ }^{2}$ UO Pediatria \\ - AO 'Ospedale San Carlo Borromeo' - Milano
}

Introduzione. Le ARI (Acute Respiratory tract Infection) sono patologie sostenute da diversi agenti eziologici in grado di causare un quadro clinico con sintomatologia a carico dell'apparato respiratorio. Gli agenti eziologici spesso associati ad infezioni delle basse vie aeree, sono i virus influenzali. Materiali e metodi : Durante la stagione influenzale 2004-2005 sono stati arruolati 100 pazienti (51 maschi e 49 femmine, età media: 19 mesi) con sintomatologia correlabile ad infezione respiratoria acuta afferenti ai servizi di Pronto Soccorso e Pediatria dell'A.O. San Carlo Borromeo di Milano. Ad ognuno di essi è stato effettuato un prelievo (aspirato naso-faringeo), sottoposto a diagnosi virologica mediante: 1) multiplex nested PCR per la simultanea rilevazione di frammenti genici codificanti per la proteina di matrice dei virus influenzali A e B; 2) multiplex nested $\mathrm{PCR}$ per l'amplificazione di frammenti genici dell'emoagglutinina dei virus influenzali di tipo A (H1 e H3); 3) analisi di sequenza del gene HA del virus influenzale di tipo A. Risultati. La ricerca di sequenze geniche dei virus influenzali A e B ha permesso di rilevare nel 12\% (12/100) dei soggetti una infezione da virus influenzale A, e nel 4\% (4/100) da virus influenzale di tipo B. La sottotipizzazione dei campioni infetti con virus influenzale A, ha evidenziato 4/12 (33.3\%) campioni positivi per $\mathrm{H} 1$ e $8 / 12$ (66.7\%) positivi per H3. Il sequenziamento genomico di campioni $\mathrm{A} / \mathrm{H} 3 \mathrm{~N} 2$ positivi ha mostrato una elevata omologia (99\%) con i ceppi virali isolati nella stagione 2004-2005 e con il ceppo vaccinale (Californialike) utilizzato in tale stagione influenzale.

Conclusioni. La diagnosi molecolare di infezione virale ha consentito di effettuare una sorveglianza virologica in tempo reale dei patogeni maggiormente correlati ad infezioni respiratorie in età pediatrica. Inoltre, l'identificazione dei match antigenici tra ceppi vaccinali ed epidemici contribuisce all'approfondimento delle dinamiche di evoluzione e di diffusione dei virus influenzali.

\section{6}

\section{ENTERITI DA ASTROVIRUS E NOROVIRUS IN ETA' PEDIATRICA: UN ANNO DI SORVEGLIANZA}

Farina C.,' Gibelli M., ${ }^{2}$ Marini F.,' Pellizzari ML.,' Podestà AF., ${ }^{2}$ Scalfaro C. ${ }^{2}$

'UO Microbiologia,

¿UO Pediatria - AO 'Ospedale San Carlo Borromeo' - Milano

Introduzione. Sebbene l'eziologia virale delle enteriti sia stata prospettata già 50 anni fa, esse costituiscono ancora oggi una patologia largamente sottostimata.

Accanto a virus il cui ruolo eziologico è stato da tempo dimostrato (Enterovirus, Adenovirus 40 e 41 e Rotavirus) soprattutto in soggetti di età pediatrica od in pazienti immunocompromessi, esistono attualmente evidenze clinico-epidemiologiche relative al coinvolgimento di altri virus, quali i Calicivirus (Norwalk-like e Sapporo-like virus), e gli Astrovirus, in episodi sporadici e/o epidemici di gastroenterite.

Materiali e metodi. I campioni fecali di 292 bimbi ricoverati presso l'UO Pediatria dell'AO 'Ospedale San Carlo Borromeo' dal marzo 2005 a febbraio 2006 sono stati esaminati per la ricerca di Salmonella spp. Shigella spp., Campylobacter spp., Rotavirus, Astrovirus e Norovirus 1 e 2. Per la ricerca di Astrovirus e di Norovirus sono stati utilizzati immunodosaggi enzimatici in fase solida: IDEIA Norovirus (Dako Cytomation Ltd, Ely, UK) ed il dosaggio immunoenzimatico amplificato qualitatitivo Amplified IDEIA Astrovirus (Dako Cytomation).

I test utilizzano un'associazione di anticorpi mono- e policlonali genere-specifici per i Norovirus di genogruppo 1 e 2 e per Astrovirus per i quali, inoltre, è prevista l'amplificazione della marcatura.

Risultati. Sono stati complessivamente identificati 9 casi (3.1\%) di infezione da Astrovirus, 4 (1.4\%) da Norovirus 1 e $6(2.1 \%)$ da Norovirus 2. I risultati sono compendiati in tabella, che riporta l'andamento stagionale dei casi osservati. \begin{tabular}{llll}
\hline Mese & Astrovirus & Norovirus 1 & Norovirus 2 \\
\hline
\end{tabular}

\begin{tabular}{llll}
\hline Marzo & - & 1 & 1 \\
\hline Aprile & 1 & 3 & - \\
\hline
\end{tabular}

\begin{tabular}{llll} 
Aprile & 1 & 3 & - \\
\hline Maggio & 4 & - & 1
\end{tabular}

\begin{tabular}{llll}
\hline Settembre & 1 & - & - \\
\hline
\end{tabular}

\begin{tabular}{llll}
\hline Dicembre & 2 & - & - \\
\hline
\end{tabular}

\begin{tabular}{llll}
\hline Gennaio & - & - & 2 \\
\hline
\end{tabular}

\begin{tabular}{llll}
\hline Febbraio & 1 & - & 4
\end{tabular}

Conclusioni. I dati del nostro Centro, per quanto ancora preliminari, sono significativi per quanto attiene alle forme sporadiche di enterite da Noro- e Astrovirus, mostrando come la circolazione di questi virus, prevalentemente inverno-primaverile (15/23 casi tra dicembre e marzo), non sia circoscritta esclusivamente ad episodi epidemici, ma possa essere descritta routinariamente nella pratica clinica. 